\title{
Squeezed-Light Optical Magnetometry
}

\author{
Florian Wolfgramm, Alessandro Cerè, Federica A. Beduini, \\ Ana Predojević, Marco Koschorreck, and Morgan W. Mitchell \\ ICFO - Institut de Ciencies Fotoniques, Mediterranean Technology Park, 08860 Castelldefels (Barcelona), Spain
}

(Dated: 25 August 2010)

\begin{abstract}
We demonstrate a light-shot-noise-limited magnetometer based on the Faraday effect in a hot unpolarized ensemble of rubidium atoms. By using off-resonant, polarization-squeezed probe light, we improve the sensitivity of the magnetometer by $3.2 \mathrm{~dB}$. The technique could improve the sensitivity of the most advanced magnetometers and quantum nondemolition measurements of atomic spin ensembles.

PACS numbers: 42.50.Lc, 07.55.Ge, 42.50.Dv, 42.65.Yj
\end{abstract}

Introduction - The ability to measure magnetic fields with high sensitivity is a key requirement in many physical, biological and medical applications. Examples can be found in the measurement of geomagnetic anomalies, magnetic fields in space as well as the measurement of biomagnetic fields such as the mapping of electric and magnetic fields produced in the brain [1 4 ].

Optical magnetometers, based on optical readout of magnetic atomic ensembles, are currently the most sensitive devices. These instruments have demonstrated sensitivities better than $1 \mathrm{fT} / \sqrt{\mathrm{Hz}}$, with rapid advancement in recent years [5 $[8]$. Two distinct sources of quantum noise determine the fundamental sensitivity of this technique: the atomic projection noise and the optical polarization noise, a manifestation of shot noise 9 12]. As today's most advanced magnetometers approach the standard quantum noise limits [13] understanding these limits becomes critical for future advances [5].

For magnetometers based on Faraday rotation and optimized for sensitivity, contributions from projection noise and light-shot noise are comparable [7, 9], and simultaneous reduction of both sources is advantageous. A pair of techniques for reducing these fundamental noise sources have been proposed, spin squeezing of the atomic ensemble 14, 15] and polarization squeezing of the probe light [9, 16], with potential to reduce the noise to the Heisenberg limit [12], except in the long-time regime where spin relaxation is limiting [9]. Recent experiments have demonstrated spin squeezing using optical quantum non-demolition (QND) measurements [17 19 and application of spin squeezing in magnetometry [13]. We report here reduction of the other fundamental noise source in optical magnetometry: we demonstrate an optical magnetometer with sensitivity better than the shot-noise limit using a polarization-squeezed probe tuned near the atomic resonance.

In other fields of optical science, the application of squeezed light has already been demonstrated, such as in polarization interferometry [20], atomic spectroscopy [21], and gravitational wave detection 22 24].

We note that the QND measurements used to produce spin squeezing are performed by the same mechanism as the spin readout, and are themselves fundamentally limited by optical shot noise [9, 11]. In that context, polarization-squeezed probing implies a greater degree of spin squeezing. Ultimately, it will therefore be desirable to employ both techniques in the same experiment [25].

The magnetometer consists of a source of polarizationsqueezed light, a rubidium vapor cell at room temperature and a shot-noise-limited polarimeter. By the Faraday effect, an axial magnetic field creates a circular birefringence in the vapor. The resulting rotation of the polarization plane of a linearly polarized input beam is seen in the detected signal. This rotation is described in terms of the probe beam Stokes parameters $S_{0}=I_{H}+I_{V}, S_{x}=I_{H}-I_{V}, S_{y}=I_{D}-I_{\bar{D}}, S_{z}=I_{R}-I_{L}$, where $I$ are the intensities of the different polarization components ( $H$ : horizontal, $V$ : vertical, $D$ : diagonal, $\bar{D}$ : antidiagonal, $R$ : right circular, $L$ : left circular). The detected signal is

$$
S_{y}^{\text {(out) }}=S_{y}^{(\text {in })}+S_{x}\left(\mathcal{V} B_{z}+\alpha F_{z}\right) l,
$$

where $\mathcal{V}$ is the Verdet constant of the vapor, $\mathbf{B}$ is the magnetic field, $\alpha$ is proportional to the vector component of the atomic polarizability, $\mathbf{F}$ is the collective atomic spin, and $l$ is the length of the medium. For a horizontally polarized probe beam, $\left\langle S_{x}\right\rangle$ is maximal and $\left\langle S_{y}^{(\text {in) }}\right\rangle$ is zero. The magnetometer signal comes from the terms $\mathcal{V} B_{z}$ and $\alpha F_{z}$, the latter being sensitive to field-induced spin precession. Projection noise is present in $F_{z}$, while shot noise is present in $S_{y}^{\text {(in) }}$. We work in a regime where these fundamental noise sources are dominant, to show clearly the advantage of squeezed light for optical magnetometry.

In one usual mode of operation, a magnetometer operates via precession of a polarized spin, the initial polarization rotating into the $z$ direction in response to the field, e.g., from $x$ toward $z$ due to $B_{y}$ as $\left\langle F_{z}\right\rangle=|F| \mu_{0} g B_{y} \tau$, where $g$ is the Landé factor, $\mu_{0}$ is the Bohr magneton, and $\tau$ is the precession time [7]. This gives a gain due to precession of $G_{y} \equiv \partial S_{y}^{\text {(out) }} / \partial B_{y}=S_{x} \alpha \mu_{0} g \tau|F| l$. Technical noise sources, e.g., in the initial orientation of $\mathbf{F}$, and environmental noise in B contribute to $\operatorname{var}\left(S_{y}\right)$ as $G_{y}^{2}$, 
i.e., as $|F|^{2}$. Similarly, $G_{z} \equiv \partial S_{y}^{\text {(out) }} / \partial B_{z}=S_{x} \mathcal{V} l$, with associated technical noise. While important progress has been made toward reducing technical and environmental noise below the quantum noise [7, 13], this is far from trivial and we adopt the simpler strategy of reducing the gain by reducing $|F|$. We work with an unpolarized ensemble, i.e., a thermal distribution within the hyperfine and Zeeman levels, with $\langle\mathbf{F}\rangle=0 . G_{y}$, the gain due to precession and the associated technical noise are then zero, while $G_{z}$ remains and we operate in the Faraday rotation mode.

The fundamental noise sources are largely unchanged in this mode of operation, and we can demonstrate shot-noise-limited performance under conditions that would be present in a highly-sensitive magnetometer with greatly reduced technical noise. The thermal distribution has intrinsic spin noise $\operatorname{var}\left(F_{z}\right)=F(F+1) N_{A} / 3$, compared to $\operatorname{var}\left(F_{z}\right)=|F| / 2=F N_{A} / 2$ for an ideal polarized state [11]. In the experiment below, the light is tuned close to the transitions from the $F=2$ manifold, which contains $5 N_{A} / 8$ atoms and for which $F(F+1) / 3=2$. The resulting spin noise detected via the last term in Eq. (11) is $\approx 5 N_{A} / 4$, versus $\approx N_{A}$ for a fully polarized $F=2$ ensemble. The shot-noise contribution is unchanged. In this way, we can see the full effects of fundamental noise sources, but with a greatly reduced sensitivity.

Experimental Setup - The experimental setup is shown schematically in Fig. 1 As principal light source we use an external-cavity diode laser at $794.7 \mathrm{~nm}$, tunable over the $\mathrm{D}_{1}$ transition of atomic rubidium. The frequency can be stabilized by FM saturated absorption spectroscopy to individual transitions of the $\mathrm{D}_{1}$ line of $\mathrm{Rb}$. The laser output passes through a tapered amplifier and is split in two parts: The weaker part is spatially filtered with a singlemode fiber and serves as local oscillator (LO) beam. The stronger part is frequency doubled to $397.4 \mathrm{~nm}$ and then sent through a single-mode fiber for mode-cleaning. Af-

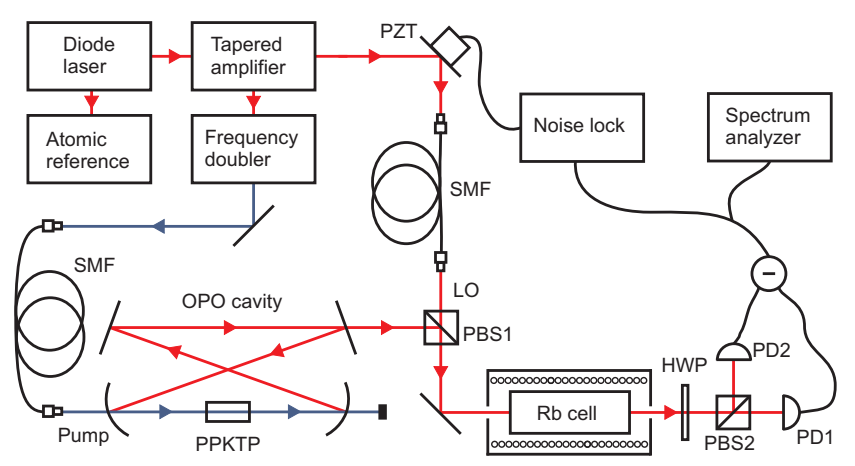

FIG. 1: Experimental apparatus. Rb cell, rubidium vapor cell with magnetic coil and magnetic shielding; OPO, optical parametric oscillator; PPKTP, phase-matched nonlinear crystal; LO, local oscillator beam; PBS, polarizing beam splitter; HWP, half-wave plate; SMF, single-mode fiber; PD, photodiode. ter the fiber a power of $42 \mathrm{~mW}$ is used to pump a subthreshold optical parametric oscillator (OPO) in which squeezed vacuum is produced. The nonlinear medium in the OPO is a type-I phase-matched PPKTP crystal. The cavity is actively stabilized by using a frequency-shifted beam with a polarization orthogonal to the polarization of the squeezed vacuum. Further details of the OPO setup can be found in [26].

The vertically-polarized cavity output is combined with the horizontally-polarized LO at a polarizing beam splitter (PBS1) with a degree of overlap of $99 \%$. The resulting light is horizontally polarized, with squeezed fluctuations in the diagonal or circular polarization basis. The polarization-squeezed light is then sent through a $15 \mathrm{~cm}$-long atomic cell at room temperature. The isotopically purified atomic vapor contains $>99 \%{ }^{87} \mathrm{Rb}$ with a small concentration of ${ }^{85} \mathrm{Rb}$. We lock the laser to the $5^{2} \mathrm{~S}_{1 / 2}(\mathrm{~F}=3) \rightarrow 5^{2} \mathrm{P}_{1 / 2}\left(\mathrm{~F}^{\prime}=2\right)$ transition of the $\mathrm{D}_{1}$ line of ${ }^{85} \mathrm{Rb}$. This corresponds to a detuning of about $700 \mathrm{MHz}$ from the closest ${ }^{87} \mathrm{Rb}$ resonance. The cell is contained within a single-layer $\mu$-metal cylinder to shield external magnetic fields while a coil within the cylinder generates the desired field $B_{z}$.

The optical rotation is detected by a shot-noise-limited polarimeter: after a half-wave plate at $22.5^{\circ}$, a polarizing beam splitter (PBS2) splits the horizontally and vertically polarized components of the beam and directs them to the two photodiodes of a balanced amplified photo-detector with a quantum efficiency of $95 \%$. The signal is monitored on a spectrum analyzer. Quantum noise locking is used to stabilize the phase of the local oscillator at maximum squeezing or anti-squeezing [27].

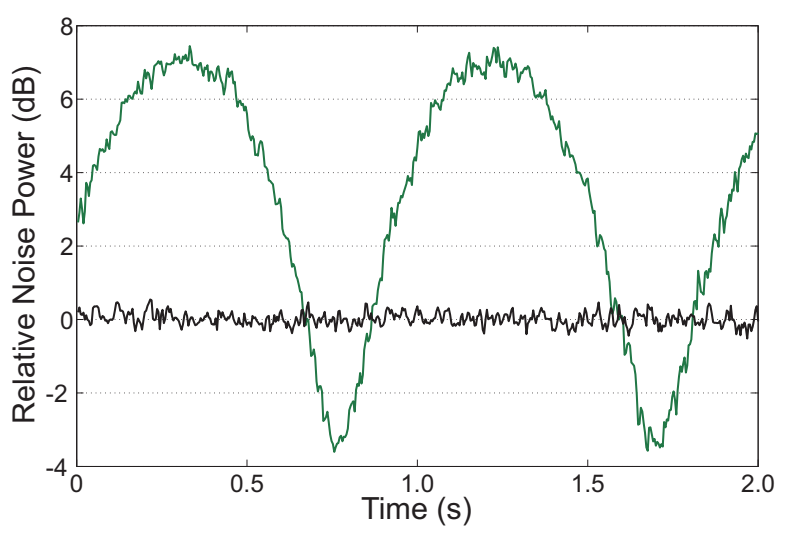

FIG. 2: Polarization squeezing after the atomic vapor cell. Polarization noise power as the phase of the local oscillator is scanned. Center frequency $1 \mathrm{MHz}$, zero-span mode, $\mathrm{RBW}=30 \mathrm{kHz}, \mathrm{VBW}=30 \mathrm{~Hz}$. Horizontal trace shows noise with a polarized (but not squeezed) probe, i.e. with OPO off, and is taken as the reference $0 \mathrm{~dB}$. Oscillating trace shows noise with $\mathrm{OPO}$ on, including regions below the shot-noise level. 
Polarization squeezing - We first characterize the polarization squeezing at the output of the vapor cell, in the absence of an applied magnetic field. The production of polarization squeezing is a phase-sensitive process, with the relative phase of the squeezed vacuum and local oscillator determining the angle of the polarization-squeezing ellipse in the $S_{y}, S_{z}$ plane [28].

The polarization noise is detected with the spectrum analyzer as the LO phase is scanned with a piezo-electric actuator, giving rise to the squeezing trace shown in Fig. 2. The electronic noise is everywhere more than $13 \mathrm{~dB}$ below the shot-noise level and is subtracted from data. The squeezing level is consistent with squeezing we observed in other measurements that were carried out without the atomic cell. The minimum of the noise level in the squeezed phase is $-3.6 \mathrm{~dB}$ below the shot-noise level and the maximum $7.4 \mathrm{~dB}$ above shot noise in the anti-squeezed phase. To our knowledge this is the highest degree of squeezing obtained in a diode-laser-pumped system.

This measurement was performed at a central frequency of $1 \mathrm{MHz}$ with zero span and a resolution bandwidth of $30 \mathrm{kHz}$, a video bandwidth of $30 \mathrm{~Hz}$ and a sweep time of $2 \mathrm{~s}$. The total detection efficiency after creation is $82 \%$ and includes the escape efficiency (96\%), the homodyne efficiency $(98 \%)$, transmission through the atomic cell (97\%) and the optical elements (95\%), and the quantum efficiency of the detector $(95 \%)$. The parametric gain, defined here as the ratio between the maximum transmission of a classical beam through the cavity with and without the presence of the co-propagating pump beam was measured to be 4.8 .

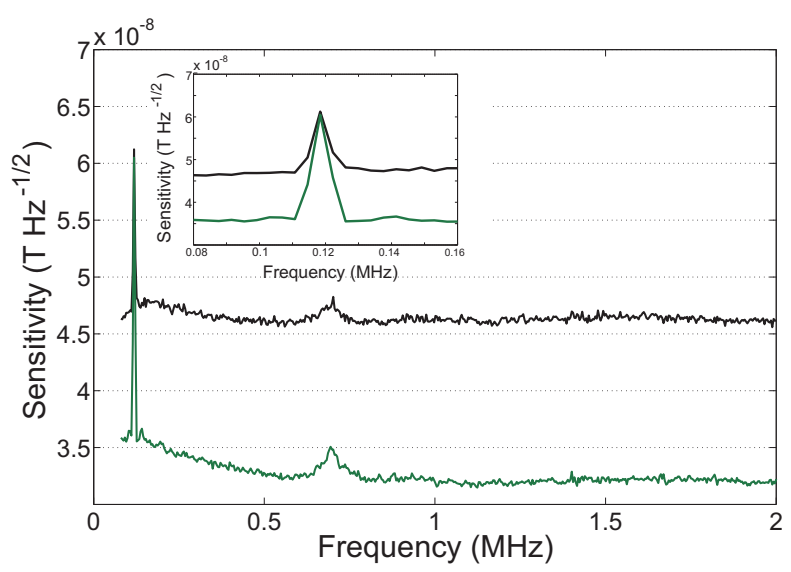

FIG. 3: Faraday rotation measurement. Power of the polarization signal as center frequency is scanned, $\mathrm{RBW}=3 \mathrm{kHz}$, $\mathrm{VBW}=30 \mathrm{~Hz}$. The (upper) black curve shows the applied magnetic signal at $120 \mathrm{kHz}$ above the shot-noise background of a polarized (but not squeezed) probe. The (lower) green line depicts the same signal with polarization-squeezing. A zoomed view around the calibration peak at $120 \mathrm{kHz}$ is shown in the inset.
Squeezing-enhanced Faraday rotation measurement - To measure the magnetometric sensitivity, we observe the Faraday rotation signal in response to an applied sinusoidal magnetic field at a frequency of $120 \mathrm{kHz}$. The sensitivity is measured with two different input polarization states: a coherent polarization state (OPO off) and a state squeezed in $S_{y}$. Quantum noise locking is used to stabilize the LO phase during the measurements. In both cases the average polarization is horizontal, due to the strong LO contribution, but the quantum fluctuations differ. As shown in Fig. 3, the observed power spectrum in both cases shows the reference signal due to the applied oscillating magnetic field at $120 \mathrm{kHz}$ above differing noise backgrounds.

The LO beam has a power of $620 \mu \mathrm{W}$ and a beam waist of $950 \mu \mathrm{m}$ inside the vapor cell. For this intensity, beam shape, and detuning, the magnetometer operates in a regime of nonlinear magneto-optical rotation (NMOR) 7]. A small fraction of the atoms are optically pumped while passing through the linearly-polarized probe beam, creating coherences within the $F=2$ manifold. Rotation of these coherences by the $z$-polarized magnetic field creates the conditions for alignment-to-orientation conversion [29 31], again by the probe beam. Measurements of rotation angle vs input power show a quadratic scaling consistent with this nonlinear mechanism. Unlike optical self-rotation [32, 33], this nonlinearity does not strongly couple optical noise into $S_{y}$, so long as the rotation angle remains small. The rotation angle was calculated to be $\phi=\left(I_{1}-I_{2}\right) /\left(I_{1}+I_{2}\right)=1.2 \mu \mathrm{rad}$, where $I_{1,2}$ are the beam intensities at the two detectors. The spectrum analyzer frequency is scanned from $80 \mathrm{kHz}$ to $2 \mathrm{MHz}$, in a sweep time of $8 \mathrm{~s}$. The resolution bandwidth and the video bandwidth were set to $3 \mathrm{kHz}$ and $30 \mathrm{~Hz}$, respectively and the signal was averaged over 130 cycles.

The polarimeter signal was calibrated against a linear magnetic field sensor inserted within the coil and shielding, thus permitting a direct conversion from measured voltage to axial magnetic field $B_{z}$. The sensitivity, i.e., field noise density as measured with the spectrum analyzer, is $4.6 \cdot 10^{-8} \mathrm{~T} / \sqrt{\mathrm{Hz}}$ for a polarized input, and reduced by $3.2 \mathrm{~dB}$ to $3.2 \cdot 10^{-8} \mathrm{~T} / \sqrt{\mathrm{Hz}}$ with a polarizationsqueezed input. It should be noted that the squeezing extends over $>2 \mathrm{MHz}$ of bandwidth, allowing magnetic field measurements in the $\mu$ s-regime with squeezingenhanced sensitivity. This technique is thus also suitable to improve $\mu$ s-scale QND measurements [11].

Conclusions - We have demonstrated the squeezingenhanced measurement of a magnetic field with a hot atomic vapor of ${ }^{87} \mathrm{Rb}$ atoms. The measurement is shotnoise-limited, and using a polarization-squeezed probe we improve the sensitivity $3.2 \mathrm{~dB}$ beyond the shot-noise level. This result complements recent demonstrations of spin squeezing to reduce spin projection noise, the other fundamental noise source in optical magnetometry. The squeezing-enhanced sensitivity extends over a bandwidth 
greater than $2 \mathrm{MHz}$, allowing high-bandwidth, sub-shotnoise magnetometry. The demonstrated technique could be applied in advanced optical magnetometers and in $\mu$ sscale QND measurements.

We are grateful to M. Napolitano and R. J. Sewell for helpful discussions and to E. Polzik for drawing our attention to Ref. [16]. This work was supported by the Spanish Ministry of Science and Innovation under the Consolider-Ingenio 2010 Project "Quantum Optical Information Technologies" and the ILUMA project (No. FIS2008-01051) and by an ICFO-OCE collaborative research program. F. W. and A. P. are supported by the Commission for Universities and Research of the Department of Innovation, Universities and Enterprises of the Catalan Government and the European Social Fund.

[1] W. F. Stuart, M. J. Usher, and S. H. Hall, Nature (London) 202, 76 (1964).

[2] M. K. Dougherty, K. K. Khurana, F. M. Neubauer, C. T. Russell, J. Saur, J. S. Leisner, and M. E. Burton, Science 311, 1406 (2006).

[3] H. Xia, A. B. Baranga, D. Hoffman, and M. V. Romalis, Appl. Phys. Lett. 89, 211104 (2006).

[4] G. Bison, N. Castagna, A. Hofer, P. Knowles, J.-L. Schenker, M. Kasprzak, H. Saudan, and A. Weis, Appl. Phys. Lett. 95, 173701 (2009).

[5] I. K. Kominis, T. W. Kornack, J. C. Allred, and M. V. Romalis, Nature (London) 422, 596 (2003).

[6] I. M. Savukov, S. J. Seltzer, M. V. Romalis, and K. L. Sauer, Phys. Rev. Lett. 95, 063004 (2005).

[7] D. Budker and M. Romalis, Nature Phys. 3, 227 (2007).

[8] G. Di Domenico, H. Saudan, G. Bison, P. Knowles, and A. Weis, Phys. Rev. A 76, 023407 (2007).

[9] M. Auzinsh, D. Budker, D. F. Kimball, S. M. Rochester, J. E. Stalnaker, A. O. Sushkov, and V. V. Yashchuk, Phys. Rev. Lett. 93, 173002 (2004).

[10] I. K. Kominis, Phys. Rev. Lett. 100, 073002 (2008).

[11] M. Koschorreck, M. Napolitano, B. Dubost, and M. W. Mitchell, Phys. Rev. Lett. 104, 093602 (2010).

[12] V. Shah, G. Vasilakis, and M. V. Romalis, Phys. Rev. Lett. 104, 013601 (2010).

[13] W. Wasilewski, K. Jensen, H. Krauter, J. J. Renema, M. V. Balabas, and E. S. Polzik, Phys. Rev. Lett. 104,
133601 (2010).

[14] A. Kuzmich, K. Mølmer, and E. S. Polzik, Phys. Rev. Lett. 79, 4782 (1997).

[15] A. Kuzmich, N. P. Bigelow, and L. Mandel, Europhys. Lett. 42, 481 (1998).

[16] D. V. Kupriyanov and I. M. Sokolov, Quantum Optics: Journal of the European Optical Society Part B 4, 55 (1992).

[17] P. J. Windpassinger, D. Oblak, U. B. Hoff, A. Louchet, J. Appel, N. Kjærgaard, and E. S. Polzik, J. Mod. Opt. 56, 1993 (2009).

[18] M. H. Schleier-Smith, I. D. Leroux, and V. Vuletić, Phys. Rev. Lett. 104, 073604 (2010).

[19] I. D. Leroux, M. H. Schleier-Smith, and V. Vuletić, Phys. Rev. Lett. 104, 073602 (2010).

[20] P. Grangier, R. E. Slusher, B. Yurke, and A. LaPorta, Phys. Rev. Lett. 59, 2153 (1987).

[21] E. S. Polzik, J. Carri, and H. J. Kimble, Phys. Rev. Lett. 68, 3020 (1992).

[22] K. McKenzie, D. A. Shaddock, D. E. McClelland, B. C. Buchler, and P. K. Lam, Phys. Rev. Lett. 88, 231102 (2002).

[23] K. McKenzie, N. Grosse, W. P. Bowen, S. E. Whitcomb, M. B. Gray, D. E. McClelland, and P. K. Lam, Phys. Rev. Lett. 93, 161105 (2004).

[24] K. Goda, O. Miyakawa, E. E. Mikhailov, S. Saraf, R. Adhikari, K. McKenzie, R. Ward, S. Vass, A. J. Weinstein, and N. Mavalvala, Nature Phys. 4, 472 (2008).

[25] J. L. Sørensen, J. Hald, and E. S. Polzik, Phys. Rev. Lett. 80, 3487 (1998).

[26] A. Predojević, Z. Zhai, J. M. Caballero, and M. W. Mitchell, Phys. Rev. A 78, 063820 (2008).

[27] K. McKenzie, E. E. Mikhailov, K. Goda, P. K. Lam, N. Grosse, M. B. Gray, N. Mavalvala, and D. E. McClelland, J. Opt. B 7, S421 (2005).

[28] W. P. Bowen, R. Schnabel, H.-A. Bachor, and P. K. Lam, Phys. Rev. Lett. 88, 093601 (2002).

[29] D. Budker, D. F. Kimball, S. M. Rochester, and V. V. Yashchuk, Phys. Rev. Lett. 85, 2088 (2000).

[30] D. Budker, W. Gawlik, D. F. Kimball, S. M. Rochester, V. V. Yashchuk, and A. Weis, Rev. Mod. Phys. 74, 1153 (2002).

[31] M. Lombardi, J. Phys. (Les Ulis, Fr.) 30, 631 (1969).

[32] M. Fleischhauer, A. B. Matsko, and M. O. Scully, Phys. Rev. A 62, 013808 (2000).

[33] J. Ries, B. Brezger, and A. I. Lvovsky, Phys. Rev. A 68, 025801 (2003). 